\title{
Detection of transit timing variations in excess of one hour in the Kepler multi-planet candidate system KOI 806 with the GTC ${ }^{\star}$
}

\author{
B. Tingley ${ }^{1,2}$, E. Palle ${ }^{1,2}$, H. Parviainen ${ }^{1,2}$, H. J. Deeg ${ }^{1,2}$, M. R. Zapatero Osorio ${ }^{3}$, A. Cabrera-Lavers ${ }^{1,2}$, \\ J. A. Belmonte ${ }^{1,2}$, P. M. Rodriguez ${ }^{1,2}$, F. Murgas ${ }^{1,2}$, and I. Ribas ${ }^{4}$ \\ ${ }^{1}$ Instituto de Astrofísica de Canarias, C/vía Láctea, s/n, 38205 La Laguna, Tenerife, Spain \\ e-mail: btingley@iac.es \\ 2 Dpto. de Astrofísica, Universidad de La Laguna, 38206 La Laguna, Tenerife, Spain \\ 3 Centro de Astrobiología (CSIC-INTA), Crta. de Ajalvir km 4, 28850 Torrejón de Ardoz, Madrid, Spain \\ ${ }^{4}$ Institut de Ciènces de 1'Espai (CSIC-IEEC), Campus UAB, Fac. de Cincies, Torre C5-parell-2a planta, 08193 Bellaterra, Spain
}

Received 13 October 2011 / Accepted 17 November 2011

\begin{abstract}
Aims. We report the detection of transit timing variations (TTVs) well in excess of one hour in the Kepler multi-planet candidate system KOI 806. This system exhibits transits consistent with three separate planets - a Super-Earth, a Jupiter, and a Saturn - lying very nearly in a 1:2:5 resonance, respectively.

Methods. We used the Kepler public data archive and observations with the Gran Telescopio de Canarias to compile the necessary photometry.

Results. For the largest candidate planet (KOI 806.02) in this system, we detected a sizable transit timing variation of $-103.5 \pm 6.9$ min against previously published ephemeris. We did not obtain a strong detection of a transit color signature consistent with a planet-sized object; however, we did not detect a color difference in transit depth, either.

Conclusions. The substantial TTV is consistent with theoretical predictions that exoplanets in resonance can produce strong transit timing variations, particularly if the orbits are eccentric. The presence of such TTVs among the bodies in this systems indicates that KOI 806 is very likely to be a planetary system. This is supported by the lack of a strong color dependence in the transit depth, which would suggest a blended eclipsing binary.
\end{abstract}

Key words. Techniques: photometric - planetary systems - stars: individual: KOI 806 - stars: individual: KIC 3832474

\section{Introduction}

Transit timing variations (TTVs) are proving to be a very valuable tool in exoplanet research. In systems with transits of different periodicities, they may serve to constrain the mass of the transiting bodies and thereby verify them as planets, without more (e.g. radial velocity) observations. Furthermore, TTVs may lead to the discovery of further non-transiting planets. Indeed, TTVs have been instrumental in characterizing multiple systems found by Kepler, including Kepler-9 (Holman et al. 2010), Kepler-11 (Lissauer et al. 2010), Kepler-16 (Doyle et al. 2011), Kepler-18 (Cochran et al. 2011), and Kepler-19 (Ballard et al. 2011), where an additional, non-transiting planet has been identified solely through TTVs.

Since its public release in Feb. 2011 of the list of Kepler planet candidates (Borucki et al. 2011, hereafter B11), the system KOI 806 has been an obvious candidate for strong TTVs. The innermost two planet candidates, designated 806.03 and 806.02, ("03" and "02" hereafter) are given with periods of $29.1654 \pm 0.0012$ days and $60.32875 \pm 0.00037$, respectively, and another candidate, 806.01 (hereafter " 01 "), is listed with $P=143.1814 \pm 0.0027$ days. The periods of 02 and 03 lie very close to the 2:1 resonance, which can strongly enhance TTVs

\footnotetext{
* Photometric data are only available at the CDS via anonymous ftp to cdsarc.u-strasbg.fr $(130.79 .128 .5)$ or via http://cdsarc.u-strasbg.fr/viz-bin/qcat?J/A+A/536/L9
}

relative to a non-resonant state (e.g. Agol et al. 2005). However, the analysis of transit timing observations from Kepler observing segments Q0-Q2 (corresponding to data up to JD 2455091.48 ) by Ford et al. (2011) stops short of listing this system among clear candidates for detected TTVs. The apparent reason is that in data from Q0-Q2, only the shortest-periodic 03 exhibited possible TTV's, whereas 01 and 02 had only one transit in these segments. Even so, the TTVs predicted by Ford et al. for all candidates in KOI 806 are among the highest of the candidates in B11; Ford et al. predict that the min-to-max TTVs over 3.5 years could range from $\sim 15 \mathrm{~min}$ for 01 to almost $5 \mathrm{~h}$ for 03 , the smallest candidate with the shortest orbit.

\section{Observations}

We used the $10.4 \mathrm{~m}$ Gran Telescopio Canarias (GTC) to observe a transit of candidate 02 with semi-simultaneous time series photometry in $g^{\prime}$ and $z^{\prime}$, two widely separated colors chromatically. These observations were undertaken in an attempt to verify that the transiting body is indeed of planetary size, because it should then show characteristic color variations caused by the interplay between the relative size of the transiting body and differential stellar limb darkening (Tingley 2004). While this detection of the color "signature" of an exoplanet can be difficult, 02 has a deep transit (almost $2 \%$ ) and a long transit duration $(6.6 \mathrm{~h})$, which made it an excellent candidate for the attempt. We obtained 


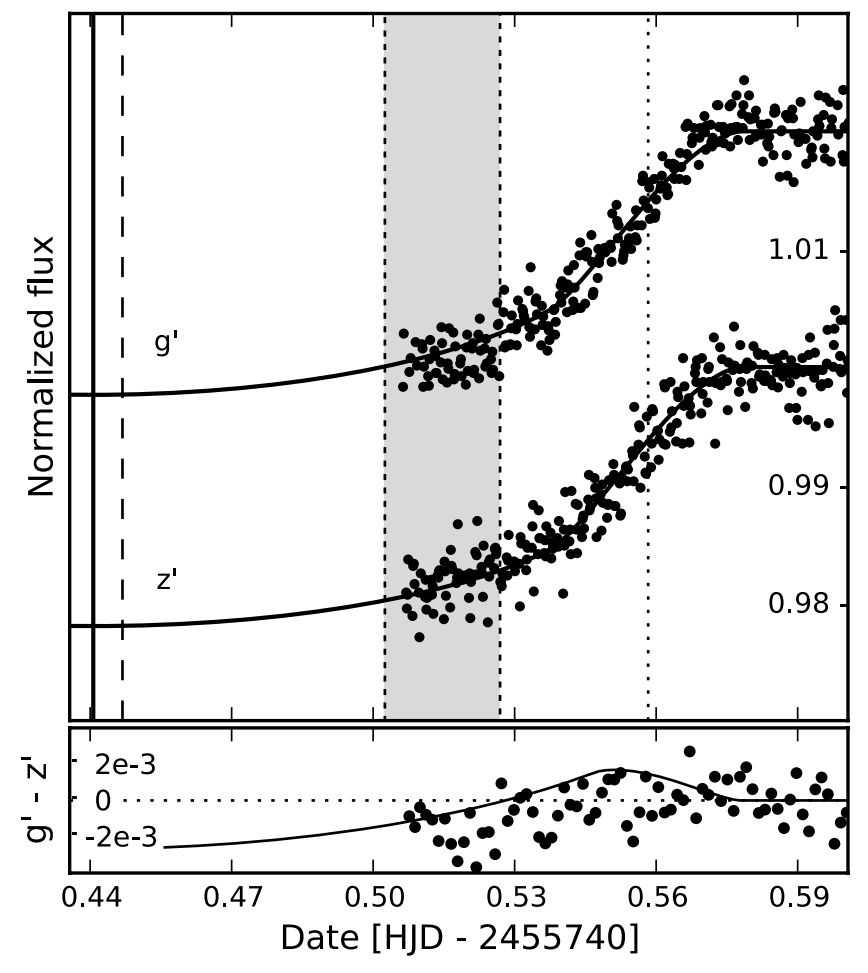

Fig. 1. GTC photometry of a transit of candidate KOI 806.02 in $g^{\prime}$ (top) and $z^{\prime}$ (bottom). The solid line is the fitted transit center and the dashed line its $3 \sigma$ confidence limit, the shaded region defines the transit center $\pm 3 \sigma$ predicted by $\mathrm{B} 11$, and the dotted line is mid-way through the egress. The time of transit center predicted by the ephemeris of B11 is $2455740.5146 \pm 4.2 \times 10^{-3}$, about $1.7 h$ earlier. The lower figure shows the observed $g^{\prime}-z^{\prime}$ during the transit egress, along with a modeled color signature using limb-darkening models from Claret \& Bloemen (2011). The points shown are the mean of each $4 \times 5 \mathrm{~s}$ exposure sequence.

approximately 1800 images with GTC/OSIRIS in five hours on the night of the 27/28th of June, 2011, alternating four 5-s exposures in $g^{\prime}(468.8 \mathrm{~nm})$ with four 5-s exposures in $z^{\prime}(893.1 \mathrm{~nm})$. Subsequent analysis using optimized aperture photometry with the "Vaphot" package (Deeg \& Doyle 2001) for IRAF showed that the transit unexpectedly ended $\approx 100$ min earlier than anticipated by the period and duration given in B11, a detection of a TTV with a significance of $\sim 15 \sigma$. Given the presence of three candidate transits in this system and the high significance of this TTV detection, it is extremely likely, just based on this, that KOI 806 harbors a planetary system of at least three planets.

In the light of this very strong TTV detection, the original science goal based on multi-color photometry becomes relegated to supporting evidence. Despite the TTV, we captured the crucial part of the egress where the planet crosses the limb of the star, which results in a weak detection of a $g^{\prime}-z^{\prime}$ color signature (on the order of one millimag), consistent with the modeled one, though offset (Fig. 1). This offset is plausible, given the clear evidence of transparency variations just after the transit, revealed by increased scatter in the photometry. This could in turn affect the measured out-of-transit flux.

However, even without a true detection of the transit color signature, we can constrain the nature of any false positive. The photometry does clearly excludes the possibility of any strong dependency of transit depth on color, which one would expect from a blend with any color difference between the components, as proposed by Tingley (2004) and used by O'Donovan et al. (2006), O'Donovan et al. (2007), Cochran et al. (2011), and Ballard et al. (2011). We derived an equation that is similar in

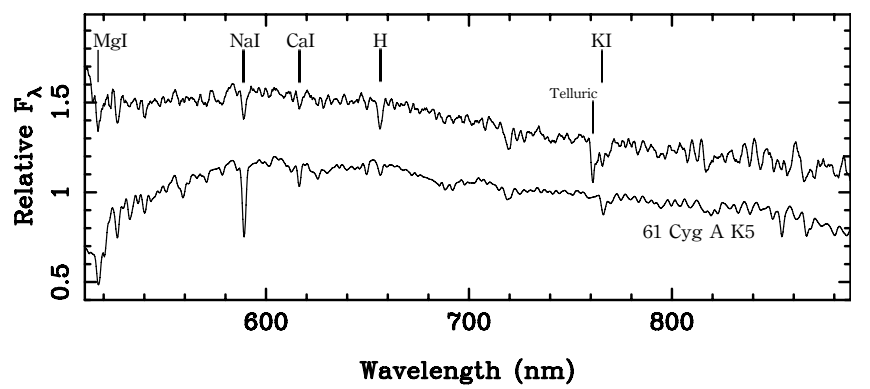

Fig. 2. ALFOSC/NOT spectrum for KOI 806 (top) along with a K5 standard star observed with the same instrumental configuration on the same night. The two spectra are smoothed by 5 pixels and offset by 0.3 for clarity, with the pertinent atomic lines indicated.

principle to BLENDER (Torres et al 2004, 2011), but much simplified, to limit the possible color difference between the target star and any contaminating eclipsing binaries (CEBs). It relates the blended eclipse depth in $z^{\prime}\left(d_{\mathrm{CEB}, g}\right)$ to that in $g^{\prime}$ using only the depth of the unblended eclipse depth in $g^{\prime}$ and $z^{\prime}\left(d_{\mathrm{EB}, g}\right.$ and $d_{\mathrm{EB}, z}$, respectively) and the $g^{\prime}-z^{\prime}$ color difference between the target star and the blending eclipsing binary $\left(\Delta\left(g^{\prime}-z^{\prime}\right)\right)$ :

$d_{\mathrm{CEB}, z}=\frac{1+d_{\mathrm{EB}, z} f_{\mathrm{EB}, z}}{1+f_{\mathrm{EB}, z}}$,

where

$f_{\mathrm{EB}, z}=\frac{1-d_{\mathrm{CEB}, g}}{d_{\mathrm{CEB}, g}-d_{\mathrm{EB}, g}} f_{\text {target }, g} 10^{0.4 \Delta(g-z)}$

where we set $f_{\text {target, } g}=1$, which yields a differential blended eclipse depth in $z^{\prime}$. If we set $d_{\mathrm{EB}, z}=d_{\mathrm{EB}, g}, \Delta\left(g^{\prime}-z^{\prime}\right)$ must be less than about 0.02 . Relaxing this constraint to allow $d_{\mathrm{EB}, z}$ to vary as much as $10 \%$ from $d_{\mathrm{EB}, q}$, a reasonable value, constrains $\Delta\left(g^{\prime}-z^{\prime}\right)$ to about 0.25 for lightly blended eclipses $\left(f_{\text {target }} \sim f_{\mathrm{EB}}\right)$ to 0.05 for highly blended eclipses $\left(f_{\text {target }} \gg f_{\mathrm{EB}}\right)$. While this does not absolutely rule out the possibility that the transit of 02 may be caused by a blend, it does suggest that it is unlikely.

While the GTC showed a clear advance of the transit egress, we would not have been able to detect any changes in transit duration because of the partial coverage of the transit. To verify the stellar parameters given in the Kepler Input Catalog for the host star, KIC $3832474\left(T_{\text {eff }}=5206 \mathrm{~K}, \log g=4.53\right)$, we took spectra of KOI 806 and several other similar stars with the $2.5 \mathrm{~m}$ Nordic Optical Telescope (NOT), using grism \#5 at a resolving power $R=830$ over the wavelength interval 5100-9000 A. The spectra were calibrated in wavelength against $\mathrm{Ne}$ lines from arc images taken with the same instrumental configuration as the target and flux normalized to unity at the wavelength interval 7400-7500 A. Uncertainty in instrumental response correction is less than $10 \%$. We corrected the spectra for telluric absorption, though some residuals at the strongest telluric oxygen band at around $7600 \AA$ remain due to different airmasses of target and telluric standard star (see Fig. 2). We obtained the spectral classification of the host star via atomic line identification (MgI, $\mathrm{NaI}, \mathrm{H} \alpha$, and $\mathrm{CaII}$ ), which indicated a spectral type of G9-K0 \pm 2 and a slightly higher temperature $\left(T_{\text {eff }} \sim 5250 \mathrm{~K}\right)$ than given in B11. We did not have the necessary spectral precision to estimate $\log g$.

This leaves the question of the radius of the star open, given by $\mathrm{B} 11$ as $R_{\star}=0.88 R_{\odot}$. A Jupiter-sized planet transiting a star of this size would cause a transit with a depth of about $1.4 \%$, whereas the observed transits of 02 have a depth of $2.0 \%$. Therefore, for the transits of 02 to be caused by a planet, 02 


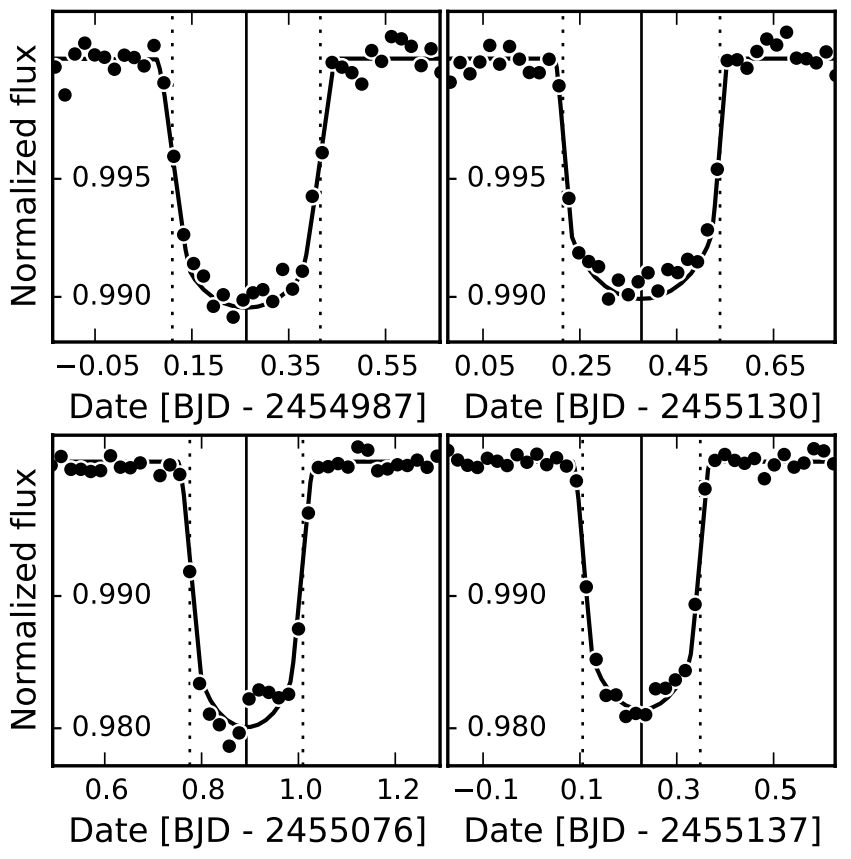

Fig. 3. Public Kepler photometry for the two released transits of KOI 806.01 (top), KOI 806.02 (bottom) along with the best-fit transits.

needs to be $\sim 20 \%$ larger than Jupiter or the star has to be smaller than expected by a similar amount - or, most likely, some combination of the two.

\section{Analysis of transits}

With the recent release of Kepler Q3 data, covering up to 16 Dec. 2009 (JD 2455182.00), we were able to extend our analysis, because second transits of both 01 and 02 are now available. We performed fits (see discussion below) to the transits in the Kepler data of each of the three candidates (see Fig. 3 for 01 and 02,03 similar but not shown).

Our parameter estimation process is based on a Bayesian methodology. We first searched for the rough global maximum likelihood solution using the differential evolution (DE) global optimization method (Price et al. 2005; Storn \& Price 1997), and then derived the parameter posterior distributions using a Markov chain Monte Carlo method (MCMC, Ford 2005). We parameterized the transits by the transit center $T_{\mathrm{c}}$, squared radius ratio $k$, period $p$, impact parameter $b$, and reciprocal of the half duration of the transit $\Upsilon$, similar to Kipping (2010a) and Bakos et al. (2010). Furthermore, we used the linear limb darkening law with a free limb darkening coefficient $u$.

We used unconstraining (uninformative) priors for the parameters estimated for the two largest candidates, 01 and 02 . For the smallest candidate, 03 , we used normal distribution priors to constrain the values allowed for $b, \Upsilon$ and $u$. The prior centers for $b$ and $\Upsilon$ were adopted from the released Kepler values and the widths were chosen conservatively, because we preferred to overestimate the errors slightly rather than underestimate them. The limb darkening prior was derived from the limb-darkening posteriors of the fits to 01 and 02 .

For the fits to the long-cadence Kepler data, we subsampled our model by 10 (i.e., each final model light curve point is an average of 10 model points uniformly spread over the time of a single Kepler exposure). This is necessary because long exposures modify the transit shape (Kipping 2010b). The Kepler

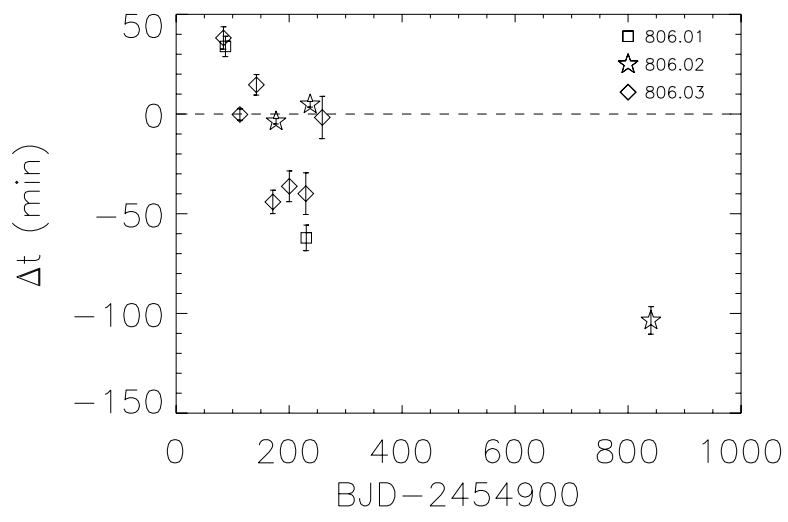

Fig. 4. Transit timing variations for the KOI 806 candidates vs. the periods and $T_{0} \mathrm{~s}$ given in $\mathrm{B} 11$. All candidates in the system exhibit high significance $(\sigma>6)$ TTVs, but KOI 806.01 and KOI 806.03 show this in only the Kepler Q0-Q3 data. KOI 806.01 has a min/max difference of over $1.5 \mathrm{~h}$ for just the first two transits, KOI $806.021 .8 \mathrm{~h}$, and KOI $806.031 .4 \mathrm{~h}$. These values are all tabulated in Table 1.

observations and the corresponding fits can be seen in Fig. 3 for 01 and 02 .

The fit to our GTC observations of candidate 02 was made simultaneously in both colors. The only major difference to the single color Kepler data is that we had separate limb-darkening coefficients for each color. Since we have only a partial transit, we cannot infer the transit duration. Instead, we used an informative prior of $5.7 \pm 0.1 \mathrm{~h}$ from the transit duration posteriors of the Kepler transit fits, and similarly, a prior for the impact parameter of $b=0.550 \pm 0.075$. The rest of the parameters have uninformative priors for conservative error estimates. The resulting residuals have a $\sigma_{\text {rms }}$ of 1.9 millimags in $g^{\prime}$ and 2.1 millimags in $z^{\prime}$ per $4 \times 5$ exposure sequence.

With the time of the transit center being one of the free fit parameters, we were able to measure the periods from the first two transits of 01 and 02 and the first seven transits of 03 , respectively, compared to the period given by B11. In combination with our own observations with the GTC, we can confirm that strong and statistically very significant TTVs are apparent in this system (Fig. 4 and Table 1).

\section{Conclusions}

We have detected strong TTVs in the KOI 806 system, affecting the timing of the transit far more than what we had expected based on the uncertainties indicated by the ephemeris of B11. The magnitude of the TTVs seen in this system are indeed not surprising, as Holman \& Murray (2005) have argued that TTVs may scale in a roughly linear fashion with period, though TTVs are highly degenerate and strongly dependent on initial conditions (Nesvorný \& Morbidelli 2008; Veras et al. 2011). If this linear relationship holds, the observed TTVs in this system are similar in scale to those observed in, for example, the Kepler-11 system (Lissauer et al. 2010) - with TTVs of tens of minutes at periods of 10-47 $d$. Our observations of 02 indicated a TTV of $-103.3 \pm 6.9$ min relative to the transit ephemeris given by the Kepler candidate list (B11). These values are based on a linear fit to all the transit that occurred during Kepler quarters Q0-Q5. Over this period of time, 02 transited five times, yet the B11 values imply a transit ephemeris error of only $\sim 4$ min for the 12th transit, the one we observed with GTC (see Fig. 1). 
Table 1. Best-fit transit times for the planet candidates KOI 806.01, KOI 806.02 and KOI 806.03, and their offset (O-C) against the ephemeris given in B11.

\begin{tabular}{lccccc}
\hline \hline$N$ & $T_{c, \text { fitted }}$ & O-C $(\min )$ & $N$ & $T_{c, \text { fitted }}$ & O-C (min) \\
\hline & KOI 806.01 & & & KOI 806.03 & \\
0 & $87.26254 \pm 5.4 \times 10^{-4}$ & $33.9 \pm 5.1$ & 0 & $83.7185 \pm 3.8 \times 10^{-3}$ & $38.2 \pm 5.6$ \\
1 & $230.37726 \pm 5.7 \times 10^{-4}$ & $-62.1 \pm 6.4$ & 1 & $112.8572 \pm 1.3 \times 10^{-3}$ & $-0.2 \pm 2.8$ \\
& & & 2 & $142.0330 \pm 2.5 \times 10^{-3}$ & $14.7 \pm 5.1$ \\
& KOI 806.02 & & 3 & $171.1576 \pm 1.7 \times 10^{-3}$ & $-44.1 \pm 5.9$ \\
0 & $176.89249 \pm 3.5 \times 10^{-4}$ & $-3.6 \pm 1.4$ & 4 & $200.3284 \pm 2.2 \times 10^{-3}$ & $-36.2 \pm 7.7$ \\
1 & $237.22714 \pm 2.3 \times 10^{-4}$ & $4.9 \pm 1.4$ & 5 & $229.4913 \pm 4.0 \times 10^{-3}$ & $-39.9 \pm 10.5$ \\
$11^{*}$ & $840.4394 \pm 2.4 \times 10^{-3}$ & $-103.5 \pm 6.9$ & 6 & $258.6832 \pm 1.2 \times 10^{-3}$ & $-1.7 \pm 10.6$ \\
\hline
\end{tabular}

Notes. $N$ is the transit number and the dates are in BJD-2 454900. ${ }^{(*)}$ From egress observed by GTC.

We performed an independent analysis of the publicly available transits (Q0-Q2), and found significant differences to the periods and $T_{0} \mathrm{~s}$ given in B11. For example, we derive a period of $60.33465 \pm 0.00042 d$ for 02 , which results in a TTV for the 12th transit of $\sim 3.2 \mathrm{~h}$. However, because many of the Q0-Q5 data are still proprietary, we cannot perform a full, independent revision of the method and results that led to the ephemeris given by B11. A TTV well in excess of one hour is certain, though, regardless of which period is used, either ours from the first two transits or that from B11. The second result of our GTC observations is the absence of any color dependency of the transit depths, the presence of which would reject the candidate, along with a color signature during egress that suggests that an object with a radii consistent with a planet is causing the transit, albeit with a low statistical significance.

While our data do not allow for a detailed analysis of the system, we consider that the existence of a very strong TTV and the absence of a color dependency of the transit depth strongly supports that the transits in KOI 806 are caused by planetary companions. Two possible scenarios for massive companions exist: either the planet is orbiting one star, or it is circumbinary. Transiting circumbinary planets have a complex pattern of transits owing to the relatively large motion of the stellar components as they orbit around the center of mass, which is clearly not observed is this system (Deeg et al. 2000; Doyle et al. 2011). Transit timing variations in systems where the planet orbits one star, called S-type orbits, are caused by the light time effect (e.g. Kopal 1959), because the distance between the transited star and the observer changes as the stars orbit around the center of mass. While this can cause high TTVs over time, a $1.7 \mathrm{~h}$ TTV requires an acceleration amounting to a change of about 13 AUs. Such a change could only be explained by some exotic, invisible and very massive object (e.g. a black hole), because a small, faint star would be unable to move the primary star so far in the less than three years over which the TTVs have been measured, and this is therefore very unlikely. Moreover, it could still not explain the TTVs measured in the other planets in the system, assuming the values in B11 are correct.

Given the long periods of the putative planets in KOI 806, it is entirely possible that this system will not be fully characterized by the end of the Kepler mission - particularly if evidence of other unknown, perhaps longer period planets arises. However, the deep transits and strong TTVs make it possible to monitor this system from ground over the long term - an endeavor likely to be scientifically worthwhile, depending on the details that emerge from the analysis of the data that are currently being acquired by the Kepler mission.
A final aspect of unknown TTVs in this and other planetary systems pertains to photometric follow-up. It appears that the transit ephemerides given in B11 do not (or cannot) necessarily account for TTVs in all cases, resulting in ephemeris errors that significantly underestimate the true timing error. Therefore, observers intending to follow up Kepler candidates, particularly in known multi-candidate systems where strong TTVs may be present, need to include this finding when planning their observations.

Acknowledgements. This article is based on observations made with the GTC operated on the island of La Palma by the IAC in the Spanish Observatorio of El Roque. The Kepler data presented in this paper were obtained from the Multimission Archive at the Space Telescope Science Institute (MAST). STScI is operated by the Association of Universities for Research in Astronomy, Inc., under NASA contract NAS5-26555. Support for MAST for non-HST data is provided by the NASA Office of Space Science via grant NNX09AF08G and by other grants and contracts. B.T. and H.D. acknowledge funding by grant AYA2010-20982-C02-02 and MRZO by grant AYA2010-21308-C03-02, both of the Spanish Ministry of Science and Innovation (MICINN). H.P. is supported by RoPACS, a Marie Curie Initial Training Network funded by the European Commission's Seventh Framework Programme.

\section{References}

Agol, E., Steffen, J., Sari, R., \& Clarkson, W. 2005, MNRAS, 359, 567

Bakos, G. Á. 2010, ApJ, 710, 1724

Ballard, S., et al. 2011, ApJ, Accepted

Borucki, W. J., Koch, D. G., Basri, G., et al. 2011, ApJ, 736, 19

Claret, A., \& Bloemen, S. 2011, A\&A, 529, 75

Cochran, W. D., Fabrycky D. C., Torres, G., et al. 2011, ApJS, 197, 7

Deeg, H. J., \& Doyle, L. R. 2001, Proc. of Third Workshop on Photometry, ed. W. Borucki, \& L. E. Lasher, NASA/CP-2000-209614, 85

Deeg, H. J., Doyle, L. R., Kozhevnikov, V. P., et al. 1998, A\&A, 338, 479

Doyle, L. R., Carter, J. A., Fabrycky, D. C., et al. 2011, Science, 333, 1602

Ford, E. B. 2005, AJ, 129, 1706

Ford, E. B., Rowe J. F., Fabrycky, D. C., et al. 2011, ApJS, 197, 2

Holman, M., \& Murray, N.W. 2005, Science, 307, 1288

Holman, M., Fabrycky, D. C., Ragozzine, D., et al. 2011, Science, 330, 51

Kipping, D. M. 2010a, MNRAS, 407, 301

Kipping, D. M. 2010b, MNRAS, 408, 1758

Kopal, Z. 1959, Close Binary Systems, The international astrophysics series

Lissauer, J., Fabrycky, D. C., Ford, E. B., et al. 2010, Nature, 470, 53

Nesvorný, F., \& Morbidelli, A. 2008, ApJ, 688, 636

O'Donovan, F. T., Charbonneau, D., Torres, G., et al. 2006, ApJ, 644, 1237

O'Donovan, F. T., Charbonneau, D., Alonso, R., et al. 2007, ApJ, 662, 658

Price, K., Storn, R., \& Lampinen, J. 2005, Differential Evolution (Berlin: Springer)

Storn, R., \& Price, K. 1997, J. Global Optimization, 11, 341

Tingley, B. 2004, A\&A, 425, 1125

Torres, G., Konacki, M., Sasselov, D. D., \& Jha, S. 2004, ApJ, 614, 979

Torres, G., Fressin, F., Batalha, N. M., et al. 2011, ApJ, 727, 24

Veras, D., Ford, E. B., \& Payne, M. J. 2011, ApJ, 727, 74 